Interim Report IR-04-042/September

\title{
Spike Trains and Kinase II for a Digital Model of Long Term Memory (An Exercise in Evolutionary Constraints)
}

Cesare Marchetti (marcheti@iiasa.ac.at)

\section{Approved by}

Leen Hordijk

Director

September 2004 


\section{Contents}

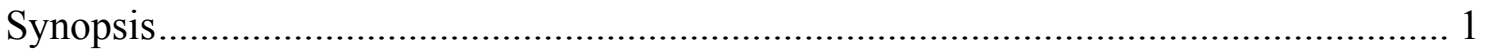

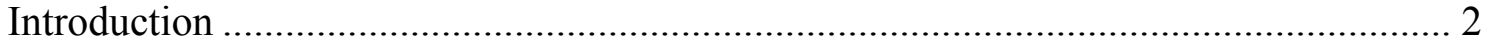

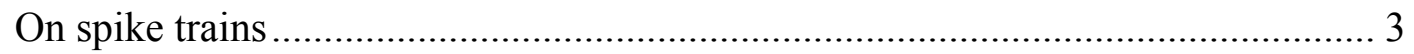

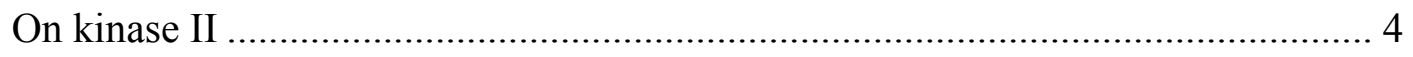

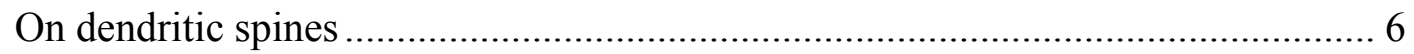

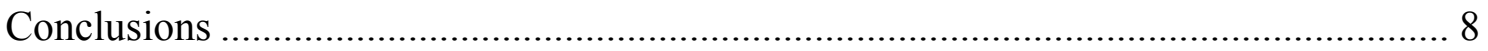

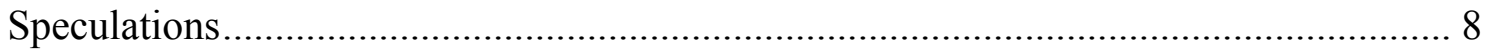

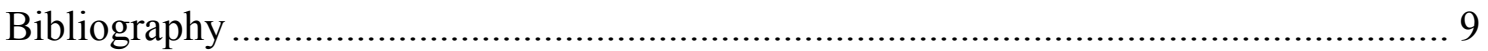




\section{Abstract}

Memory is the repetition of an event and this requires re-firing a sufficient number of neurons that did participate to the event.

We assume that an event is identified by a digital code sealed into a molecular counter, kinase II, capable of taking billions of configurations.

We also assume that spike trains represent numbers in binary notation that are able to code and decode kinase II.

Coding means that a number is imprinted by setting kinase II in a certain configuration, decoding means activating kinase II carrying that number in order to liberate $\mathrm{Ca} 2+$ via Calmodulin and open the various segments of circuit where it is located.

We assume that coded kinase II is stored in dendritic spines, adjacent to synapses, that protect it from metabolic destruction of the coding.

The model easily interprets the fact that the same optical or olfactory stimuli will induce identical spike trains.

Because identification requires the re-opening of a specific memory.

So the smell of a rose must open the circuit of the rosesmell via the appropriate spike.

Keywords: long term memory, spike trains, dendritic spines, kinase II 


\section{Acknowledgment}

The Sloan Foundation of New York for financial support in the course of this study. 


\section{About the Author}

Cesare Marchetti started his career as a physicists, was then manager of research laboratories, and finally spent 30 years as a researcher in IIASA doing system analysis. He produced very simple predictive models, the physicist way, on the most variegated subjects, from the dynamics of energy markets to the evolution of transport systems. His papers are collected on his web site www.cesaremarchetti.org/. 


\section{Spike Trains and Kinase II for a Digital Model of Long Term Memory (An Exercise in Evolutionary Constraints)}

Cesare Marchetti

\section{Synopsis}

The hypothesis that spike trains are digital signals representing binary numbers (Softky 1995) is taken as correct. Because the spikes are precisely timed inside the train(Fig 1), it can be assumed that the time slots containing a spike represent 1 and those not containing a spike represent 0 . The numbers represented by an actual spike train with such binary coding may be then very large, in the trillion range.

Kinase II is a composite molecule made of 8 or 10 or 12 modules each capable to phosphorylate on three positions. The total number of different configurations the molecule can assume happens to be in the range of trillions (Coomber b,1998). The modules interact in a ratcheting mode that makes the molecule a suspect digital counter. Kinase II is usually associated with the calcium carrier Calmodulin providing a good link between activation and action via the liberation of $\mathrm{Ca} 2+$.

Dendritic spines are microscopic structures that form in the hundreds on the surface of dendrites. In spite of their very small size they are highly structured and very active. They are linked to the dendrite with necks of various shapes which adumbrate gating functions.

Various combinations were tried to convey the complexity of the components toward doing useful work. On the evolutionary argument that complexity will not be preserved if it doesn't have an appropriate job to perform.

The simplest and intuitively most plausible model that could be patched up is given in the following and provides interesting outlines for a possible mechanism of long term memory. The wording explicitly assumes this possibility.

1) kinase II may be coded into a certain configuration by a spike train carrying a number presented in synchrony with an event in which the kinase is involved being physically present in the space of the event.. The number could be produced e.g., by a central clock, the spike train being the carrier of the coding signal over the brain.

2) kinase II could be decoded ie activated by a spike train carrying the same number that served to code it. This means kinase II may operate as a counter with a trillion different positions that could 'remember' a given one. The activation should lead to a mobilization of $\mathrm{Ca} 2+$ via the cofactor Calmodulin. 
3) In order to serve for memory preservation, coded kinase II must be protected from further imprinting and dismantling. Here the spines may come to help. Their protected and protective structure may defend kinase II from re-coding and from metabolic destruction. A tall order but inevitable in any long term memory mechanism.

4) When a spike train carrying the same number that originally coded our kinase II roams the brain, then our kinase II are reactivated and stimulate their associated co-factor calmodulin to release $\mathrm{Ca} 2+$ that can initiate a chain putting into action a synapse next to the spine or on the spine itself.

In summary, the physical seat of long-term memory could then be in the spines that cover the surface of each dendrite by the hundreds, each spine holding kinase II counters that may freeze the reference number of an event. The wrapping of the counters in a protected environment that make them survive the life of an individual has analogies with the formation of spores and hints at processes of fixation that may well require the intervention of DNA.

A recall is the repeat of an event meaning that a sufficient number of neurons that participated to the original event must fire again to recreate the memory of the event itself. In a configuration where a set of spines, be them a million, holds kinase counters set on the same number, the neuronal topography of an event is defined in terms of the dendrites holding these spines. A spike train switching them into action produce the rehearsal of the event, ie the memory recall.

If sufficient family resemblance exists between kinase II and kinases $\mathrm{C}$ in general, one may speculate that kinases $\mathrm{C}$, which mediate action in a vast number of events, may be activated by similar procedures. This view would suggest central controllers dispatching their orders all over the organism in digital form .

As Darwin teaches a successful mechanism not only is conserved and evolved, but it's application tends to diffuse where it is successfully applicable.

Because these result, if hypothetical, may finally help to find guidelines to disentangle the unsolved problem of long term memory, it could be useful to detail on the subject.

The model at least would fit by a quantitative fit the Darwinian constraint that the complexity of these brain components should correctly match the complexity of their function.

\section{Introduction}

Long-term memory stores an immense amount of information that brain machinery retrieves almost instantly and, in most cases, with crystal clarity even tens of years after the event. A satisfactory account of the mechanisms for storage and retrieval has remained elusive although quantitative considerations make inevitable that memorization is at the molecular level.

Consider that a recall is not a shorthand for an event. Rather, it is the repetition of the event by a representative number of neurons that originally participated in it. These neurons constitute an extremely complex web of interconnections, based 
principally on the dendrites, which plug into other neurons through nonlinear switches, the synapses.

A recall then requires then the simultaneous switching on of thousands of synapses by a signal of some sort that travels through the brain.

Because a given synapse may have participated in many different events, $a$ specific coding for each event must be preserved in all of them to reset specifically that particular circuit. The current consensus on the mechanism for long-term memory, long term synaptic potentiation or LTP, meaning that a past event makes a synapse easier to open, is at least incomplete, because it lacks the necessary specificity.

By general consensus, three brain components: spike trains, kinase II, and dendritic spines, appear important in the operation of the long term memory system because they always seem to be present and knockout mutants show evident deficits. Their functions in the memory process however are insufficiently identified.

In order to fit the tenet that complexity in structure must match complexity in function a model has been constructed that could provide the specificity needed for synchronized synapses opening and more generally for a theory of long-term memory.

The basic tool used to select consistent hypotheses is the Occam's Razor of Darwinian theory. In short, as said before, a complex machinery cannot survive, for long, the wear and tear of stochastic evolution if it is not fully and proficiently employed.

\section{On spike trains}

The first element in the process is the spike train, a brief sequence of voltage pulses or action potentials that may take hundreds of milliseconds. Inside the train the pulses are precisely positioned into time slots, where a pulse may be present or not (Fig.1). The irregular distribution of pulses shown in the figure is more apparent than real, since the discovery that the pulses fall precisely into individual time slots having a width of the order of ten millisecond with a spread of the order of a millisecond.

That spike trains represent numbers, presumably in binary notation, is relatively well accepted in spite of the seminal work of Softky. If we visualize the slots containing a pulse as 1's and the slots with no pulse as 0's the spike train appears as a straight binary digit, incidentally a large one representing an order of trillions. In spite of the work of Softky and others however the function of these numbers roaming the cortex remains mysterious.

A modern and controversial view is that the timing of the pulses carries information, either intrinsically or in relation to the timing of other spike trains. Softky (National Institutes of Health, Bethesda MD) appears one of the most enthusiastic and articulate propagators of the idea that these sequences encode binary digits and are used by the brain to perform its internal calculations.

Softky $(1994,1995,1996)$ extensively and convincingly demonstrate that a digital system of computation in the brain would be vastly superior to an analogue system. However, he could not show how the brain actually performs its calculations using spike trains. 
The suspicion that the spike trains may carry messages to open a gate in a lock and key configuration is shown by the fact that in the sensory system of insects (locusts, to mention a well-studied case) olfactory sensors send their messages to the brain in form of spike trains. To a given "simple" odor, call it rose or turpentine, always corresponds the same spike train. One simple odor, one typical spike train.

If the spike train is the key that opens the appropriate set of locks identifying e.g. the circuits that fired during the first experience of a rose scent, then every time that spike train arrives, that memory of rose scent will be reenacted by reopening the appropriate neuronal circuits.

The eye also communicates to the brain via spike trains. Here the input is more variegated, and it is hard to reach the point simplicity of a single odor. Moreover, one cannot expect that a given visual image is already in store, to be reenacted as a memory when the spike train signal reaches the brain. However, many experiments show the same spike train output for the same sensory input.

It may be interesting to observe that a single projection neuron can respond to different odors with distinct temporal pattern of spike trains. This points to the fact spike trains of a given structure may be started from a single coded molecule or a group of them inside a single cell opening the possibility of coded kinase II sittig upstream of spike trains.

From Darwinian considerations alone monocellular organisms must be endowed with elaborated and articulated computing systems. This is very apparent if we examine the complex behavior eg of protozoa as explicitly stated eg by Csaba.

A most interesting feature of spike trains is that a neuron can reproduces exactly the same pattern of impulses with accuracy better than a millisecond.

Spike trains are well-conserved in animals having a nervous system, be they human, mouse, or locust. All the important details are conserved, e.g., the precise location of the spikes in a time slot reference system. Inevitably spike trains must be central to brain operation.

A spike train would be central if this is the way a given neuronal event becomes digitized, so that the memory of this event can be reactivated by a number carried by spike train roaming the brain as it is proposed in our model.

Because the spike train can be the digital key we must identify a digital lock with a comparable number of positions. The candidate number one is kinase II that can take a very comparable number of different configurations, as said in the order of trillions.

It must be clear that this statement descends from Darwinian necessity only. A thorough search of the literature didn't show any research done on a possible interaction, if mediated in complex ways, between spike trains and kinase II.

\section{On kinase II}

Kinase II belongs to the ubiquitous class of protein kinases, important in managing all sort of processes inside an organism. Kinase II is a modular molecule made up of a set of subunits. In the brain it may constitute up to $30 \%$ of the proteins in the space near the synapse, and with a "smoking gun" argument, researchers saw it as the molecule of 
memory. It is normally associated in a complex with the calcium carrier calmodulin and is coded then as CaMKII.

Kanaseki et al. (1991) published excellent observations of kinase II by electron microscopy. The molecule is bulky, so that the images come out well defined (Fig.1). A set of more or less similar modules, 8,10 , or 12, arranged in the form of a rosette, make up the molecule.

Each module has the form of a dumbbell, with a small ball and a large one. The small balls coalesce around a central hole and the large ones spread out. Each module can phosphorylate at three sites. This phosphorylation and its stability depend on the state of neighboring components.

This is the basis for a ratcheting process that would be necessary for a counter.

Looking at the molecules in Kanaseki's pictures, one can't resist thinking that kinase II must be a counter of some sort. The interaction between the modules could provide the ratcheting mechanism (e.g., through sequential phosphorylation) that would make possible to exploit its large capacity for different configurations in an ordered manner.

That capacity depends on how the counter is operated. Coomber (1998b) in a paper presented by J. Lisman of Brandeis, a great supporter of CaMKII as a memory molecule, calculates $10^{12}$ possible configurations for the 10 -unit version of kinaseII that seems most common in the brain.

Such a complex machine, probably present in all nervous systems and highly conserved, would certainly be evolutionarily degraded if its full potential were not central to the functioning of the brain. In spite of this evolutionary constraint, researchers who came to the idea that kinase II might be a switch never went beyond the hypothesis that it is a binary switch (Lisman, 1994; Matsushita et al., 1995), or a frequency detector (Hanson et al., 1994), or a recorder of $\mathrm{Ca} 2+$ transients spacings (Dosemeci and Albers, 1996).

Coomber, bringing skills in computers and informatics, best analyzed kinase II in physical and functional terms and cleverly dug into the gears of the machine (Coomber, 1998). Coomber did not spell out the word counter, although he spoke of a binary switch, perhaps a graded one. The definition of graded switch is certainly acceptable if only one could contemplate one trillion grades.

To use its configurational potential of $10^{12}$ states (for the 10 -subunit molecule), it seems inevitable that kinase II be a counter with $10^{12}$ positions or so. The conservation argument is a fairly hard proof for this hypothesis although other hypotheses in the functioning might be possible. Only experimentation can here decide.

Certainly one has to test the counter with appropriate signals for inputs and outputs. If this hypothesis is correct, only sophisticated time structures such as spike trains could do that work. Experiments have been tried applying oscillatory signals at constant frequency to kinase II. These experiments may imitate too roughly what happens with spike trains, so the reactions, are mixed and confused. But there are reactions.

Kinase II usually operates in connection with a cofactor, calmodulin, which appears to be a universal calcium carrier. It normally holds 4 calcium atoms. Calcium 
$(\mathrm{Ca} 2+)$ on the other side is the universal signal for action in biological systems Ion pumps keep it in low concentration in the cytoplasm, so that a raise of $\mathrm{Ca} 2+$ is interpreted by cellular machinery as a signal to operate, contracting a muscle, opening a synapse, or reading DNA.

Because of their fixed association kinase II and calmodulin are usually coded as CaMKII. But in this paper they have been kept separate as the quintessential information processing seems to be concentrated in kinase II and it's structure.

In sum, the second and central hypothesis for the functioning of memory is that kinase II is a counter that can be preset to a given number (configuration) and later reactivated. More specifically, a spike train representing a digital number sets kinase II into one of its $10^{12}$ states and a spike train carrying the same number, re-activates it in terms of stimulating calmodulin to liberate $\mathrm{Ca} 2+$.

\section{On dendritic spines}

The nervous system preserves its complexity even in its finest recesses. There are, for example, two internal spaces to the cell, the cytoplasmatic (the normal one for a cell) and the endoplasmatic reticulum. The endoplasmatic reticulum, which keeps and provides ions for the working of the neuron, penetrates as an independent structure the entire neuron including the dendrites, down to the final recesses, the dendritic spines, (Figure 1).

Dendrites are very thin, in the micron range, and provide terminal and intermediate synaptic connections. On their surface they carry small protuberances, the dendritic spines, our third component, . Well-examined with electron microscopy and two photon microscopy, the dendritic spines show a range of shapes, from a flat waddle to a pear with a thin neck.

Dendritic spines contain all the machinery for neuronal operation, in particular CaMKII, which represents their largest protein constituent. They are also penetrated by the endoplasmatic reticulum, the universal tool bag of the neuron machinery. Because the dendritic spines are always implicated with memory, in one way or another, any "smoking gun" theory must also give them great importance in memory processes.

It must be kept in mind that the neck that joins the spine to the dendrite, even if narrow, does not deny access of spine fluids to the body of the dendrite and vice versa. The neck may also shut out the fluid, so that the spine could be seen as a gated recess of the dendrite itself. The tall order of protecting coded kinase II from recoding and metabolic destruction however is again a Darwinian requirement.

Dendritic spines have been quite thoroughly studied during the last twenty years with the rise of techniques that overcome the problem of their very small size. For example, two photon microscopy and laser microscopy permit views of the spines when functioning.

The third element in the model then is that the physical seat of long term memory is in the dendritic spines each of them holding a number of kinaseII moleculescounters that can "freeze" the reference number of an event by taking and holding the corresponding configuration. 

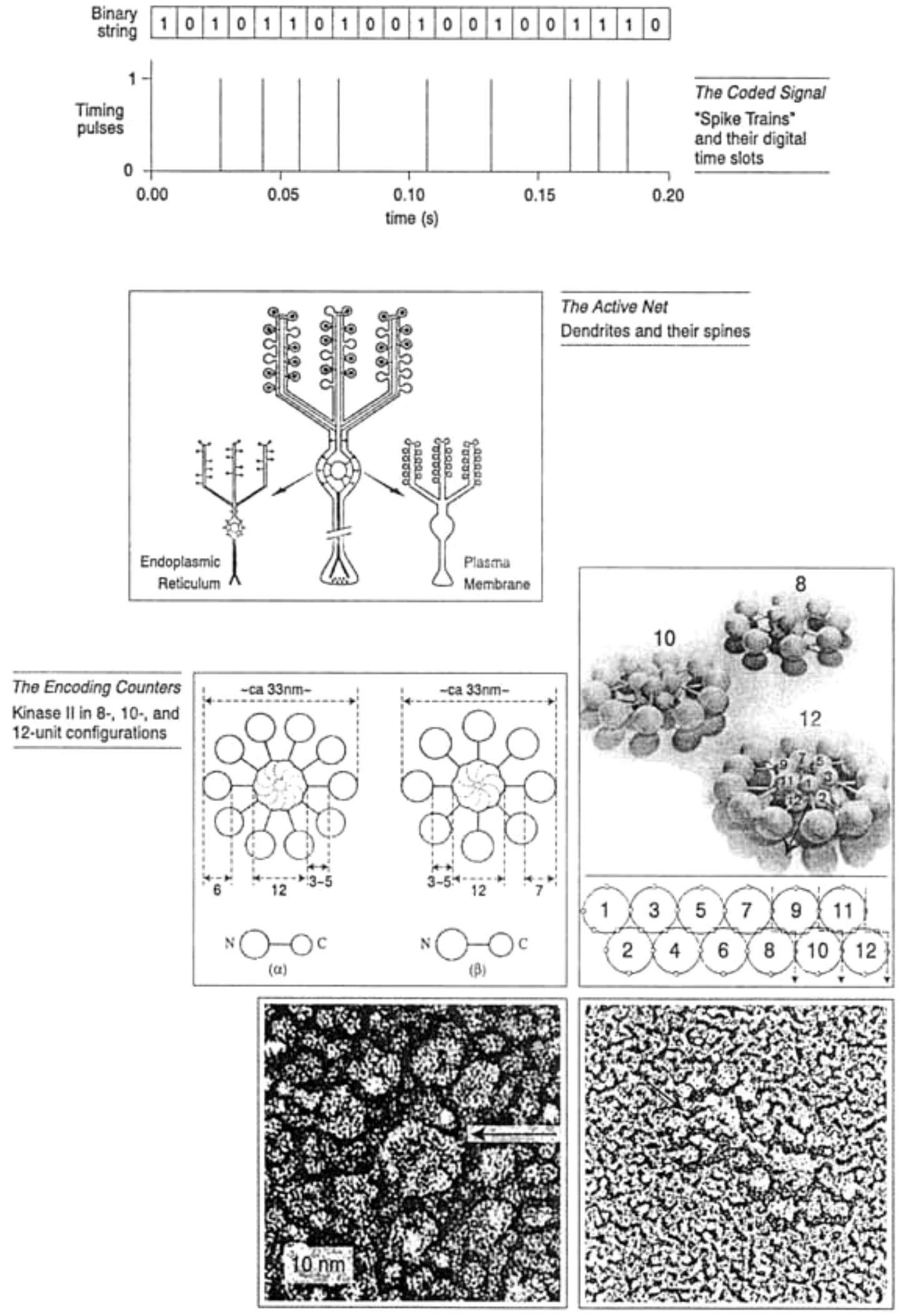


\section{Conclusions}

The leading idea has been that spike trains, kinase II, and dendritic spines are complex, widespread, and conserved systems and consequently must have a general, important, and sufficiently complex task to perform.

Spike trains and kinase II can be seen as representing numbers, very large ones and of similar magnitude, and thus it has been tried to make them work together numerically at full performance, as keys and locks, by attributing to spike trains the task of carrying digital gate signals in binary form, and to kinase II the task of a digital gate that can be set on and unlocked by spike trains.

Once this basic mechanism is accepted, if hypothetically, then the description of the functioning of memory becomes quite straightforward.

Remembering means re-enacting an event at the neuronal level, and this requires opening a large set of synapses to reactivate the original circuits that fired when the event first did happen. If a specific event is dubbed via a specific number stored in dendritic spines near these synapses or carrying synapses themselves, then the reactivation of kinase molecules carrying that same number could do the job.

Activated CaM KII can liberate $\mathrm{Ca} 2+$ that can activate the synapses nearby.

Coded kinase II molecules should be better preserved in a protective receptacle ready for use. Dendritic spines are these candidate receptacles, tiny, sophisticated, and conserved structures whose complex machinery would not resist evolutionary wear and tear if their role were not essential.

In the model their role is in fact very complex. They have to produce or gather coded kinase II, preserve it against metabolic destruction for the life of the individual, make its reactivation easy and fast, and signal for the opening of the synapse when the appropriate spike train comes.

The process of memory could then be described as basic four steps:

1) coding of kinase II into a certain configuration via a spike train;

2) conservation of a certain amount of the coded kinase II into a spine;

3) activation of this kinase II and the associated calmodulin with a spike train carrying the appropriate signal

4) starting a $\mathrm{Ca} 2+$ activated process from the spine, activating a nearby synapse.

The common problem to all models of long term memory, how to skip molecular destruction by metabolic processes is skipped here by assigning a hypothetically protected space inside the spines. The search for the mechanism is open.

\section{Speculations}

The obviously complementary problem is who and how starts the chain of events leading to a recall. This problem has not been touched because the scanning of the literature did not reveal any element that could be used even for hints.

However, evolution again imposes economy and ingenuity. When a trick is invented, it will finally find its place whenever it can be used. Kinase II as a counter 
with $10^{12}$ positions (or whatever it may be in practice) is a sophisticated engine. It would be natural to use it also uphill, to help code the spike train that, downhill, will reopen the neuron chain related to a given event. The fact that spike trains in the olfactory system may be started in a single cell shows that one or more gated molecular counters can be located just in a single cell and operate as starters.

This leads to the next question: Even if the information is there, how it is set properly in motion? Protozoa swimming in a drop of water react to disturbances in ways reminiscent of a dog or a hen, showing they must have computational machinery and memory.

Eminent students of protozoa, such as Csaba (Csaba and Kovacs, 1986), have stated this view explicitly, but nobody seems to have tried to see how the machinery works in detail.

In terms of evolution, Darwin would certainly state that cells making the brain did not shed their computing and remembering capacity, but presumably interconnected it in a network, or more precisely a web, as each "minicomputer" can connect up to say 100,000 other ones.

If that reasoning is correct, a single cell may hold the code of a single memory in the form of one or more molecules of coded kinase II and be able to initiate the spike train that will open the circuit.

Protection from metabolic destruction is not a desperate problem. Prion molecules have configurations that are not metabolized. And incidentally prions are present in brain and are supposed to have important functions there as knockout animals have shown.

As a further thought, kinases $\mathrm{C}$, a large and omnipresent family of actuators, are mostly made by a smaller number of modules, e.g., three. Once the principle of the digital chain of command is established, why not extend it to the peripheral outposts?

In these outposts the commands can employ much shorter arithmetics, as the alternative decisions are less numerous and consequently the counters can have a smaller capacity.

This outlines the picture of an organism where the management runs by numbers, fitting well the general picture where the basic mechanisms of life are digitized, starting from DNA.

Darwin would certainly like that.

\section{Bibliography}

Bailey, C.H., Bartsch, D., and Kandel, E.R., 1996, Toward a molecular definition of long-term memory storage, Proc. Natl. Acad. Sci. USA, 93:13445-13452.

Bargmann, C.I., 1998, Neurobiology of the Caenorhabditis elegans genome, Science, 282:2028.

Barnes, C.A., 1995, Involvement of LTP in memory: Are we "searching under the street light"? Neuron, 15:751-754. 
Bayer, K.U., Harbers, K., and Schulman, H., 1998, $\alpha$ KAP is an anchoring protein for a novel CaM kinase II isoform in skeletal muscle, EMBO Journal, 17(19):55985605.

Berridge, M.J., 1997, Elementary and global aspects of calcium signalling, Journal of Physiology, 499(2):291-306.

Berridge, M.J., 1998, Neuronal calcium signaling, Neuron, 21:13-26.

Bootman, M.D., and Berridge, M.J., 1995, The elemental principles of calcium signaling, Cell, 83:675-678.

Cherkashin, A.N., and Azarashvili, A.A., 1972, Pharmacological studies of memory, Soviet Neurology and Psychiatry Journal, 22(3):504-509.

Coomber, C., 1998a, Current theories of neuronal information processing performed by $\mathrm{Ca} 2+/$ calmodulin-dependent protein kinase II with support and insights from computer modelling and simulation, Computers Chem., 22(2-3):251-263.

Coomber, C.J., 1998b, Site-selective autophosphorylation of $\mathrm{Ca} 2+/$ calmodulindependent protein kinase II as a synaptic encoding mechanism, Neural Comput. 10(7):1653-78.

Covey, E. and Casseday, J.H., 1991, The monaural nuclei of the lateral lemniscus in an echolocating bat: Parallel pathways for analyzing temporal features of sound, Journal of Neuroscience, 11(11):3456-3470.

Csaba, G., and Kovacs, P., 1986, Studies into disturbing receptor "memory" in a unicellular (tetrahymena) model system: Changes in the imprinting potential on exposure to combinations of related and unrelated hormones, Expl. Cell Biol., 54:333-337.

De Koninck, P., and Schulman, H., 1998, Sensitivity of CaM kinase II to the frequency of Ca2+ oscillations, Science, 279:227.

Dosemeci, A., and Albers, R.W., 1996, A mechanism for synaptic frequency detection through autosphorylation of CaM kinase II, Biophysical Journal, 70:2493-2501.

Goldman-Rakic, P.S., 1996, Memory: Recording experience in cells and circuits: Diversity in memory research, Proc. Natl. Acad. Sci, USA, 93:13435-13437.

Hanson, P.I., and Schulman, H., 1992, Neuronal Ca2+/calmodulin-dependent protein kinases, Annu. Rev. Biochem., 61:559-601.

Hanson, P.I., Meyer, T., Stryer, L., and Schulman, H., 1994, Dual role of calmodulin in autophosphorylation of multifunctional $\mathrm{CaM}$ kinase may underlie decoding of calcium signals, Neuron, 12:943-956.

Hardingham, G.E., Sangeeta, C., Johnson, C.M., and Bading, H., 1997, Distinct functions of nuclear and cytoplasmic calcium in the control of gene expression, Nature, 385:260-265.

Hendrix, R.W., Smith, M.C.M., Burns, R.N., Ford, M.E., and Hatfull, G.F., 1999, Evolutionary relationships among diverse bacteriophages and prophages: All the world's a phage, Proc. NAS, 96(5):2192-2197.

Jeffery, K.J., 1997, LTP and spatial learning - Where to next? Hippocampus, 7:95-110. 
Jensen, O., and Lisman, J.E., 1996a, Hippocampal CA3 region predicts memory sequences: Accounting for the phase precession of place cells, Learning \& Memory, 3:279-287.

Jensen, O., and Lisman, J.E., 1996b, Theta/gamma networks with slow NMDA channels learn sequences and encode episodic memory: Role of NMDA channels in recall, Learning \& Memory, 3:264-278.

Joerges, J., Kuettner, A., Galizia, G., and Menzel, R., 1997, Representations of odours and odour mixtures visualized in the honeybee brain, Nature, 387:285.

Kanaseki, T., Ikeuchi, Y., Sugiura, H., and Yamauchi, T., 1991, Structural features of $\mathrm{Ca} 2+/$ calmodulin-dependent protein kinase II revealed by electron microscopy, Journal of Cell Biology, 115(4):1049-1060.

Katz, L.C., and Shatz, C.J., 1996, Synaptic activity and the construction of cortical circuits, Science, 274:1133.

Koester, H.J., Sakmann, B., 1998, Calcium dynamics in single spines during coincident pre- and postsynaptic activity depend on relative timing of back-propagating action potentials and subthreshold excitatory postsynaptic potentials, Proc. Natl. Acad. Sci. USA, 95:9596-9601.

Laurent, G., Wehr, M., Davidowitz, H. J., 1996, Temporal representations of odors in an olfactory network, Neuroscience, 16(12):3837-47.

Lisman, J., 1994, The CaM kinase II hypothesis for the storage of synaptic memory, Trends Neurosci, 17(10):406-412.

Lisman, J.E., 1997, Bursts as a unit of neural inforamation: Making unreliable synapses reliable, Trends Neurosci., 20(1):38-43.

Lisman, J.E., 1999, Relating hippocampal circuitry to function: Recall of memory sequences by reciprocal dentate-CA3 interactions, Neuron, 22:233-242.

MacLeod K., Backer A., and Laurent, G., 1998, Who reads temporal information contained across synchronized and oscillatory spike trains? Nature 395:693-698.

Mainen, Z.F., Joerges, J., Huguenard, J.R., and Sejnowski, T.J., 1995, A model of spike initiation in neocortical pyramidal neurons, Neuron, 15:1427-1439.

Matsushita T., Moriyama S., Fukai T., 1995, Switching dynamics and the transient memory storage in a model enzyme network involving $\mathrm{Ca} 2+/$ calmodulindependent protein kinase II in synapses, Biol. Cybern. 72(6):497-509.

Moortgat, K.T., Keller, C.H., Bullock, T.H., and Sejnowski, T.J., 1998, Submicrosecond pacemaker precision is behaviorally modulated: The gymnotiform electromotor pathway, Proc. Natl. Acad. Sci. USA, 95:4684-4689.

Mons, N., Guillou, J.-L., and Jaffard, R., 1999, The role of Ca2+/calmodulin-stimulable adenylyl cyclases as molecular coincidence detectors in memory formation, Cellular and Molecular Life Sciences, 55:525-533.

Morrison, G.E., Wen, J.Y.M., Runciman, S., and van der Kooy, D., 1999, Olfactory associative learning in Caenorhabditis elegans is impaired in lrn-1 and lrn-2 mutants, 
Behavioral Neuroscience, 113(2):358-367.

Neher, E., and Sakmann, B., 1992, The patch clamp technique, Scientific American, March:28.

Nicolelis, M.A.L., Baccala, L.A., Lin, R.C.S., and Chapin, J.K., 1995, Sensorimotor encoding by synchronous neural ensemble activity at multiple levels of the somatosensory system, Science, 268:1353.

Rieke, F., Warland, D., de Ruyter van Steveninck, R., and Bialek, W.,1996, Spikes: Exploring the Neural Code, MIT, Cambridge, MA, USA.

Silva L.R., Amitai Y., Connors B.W., 1991, Intrinsic oscillations of neocortex generated by layer 5 pyramidal neurons, Science, 251:432-5.

Singer, W., and Gray, C.M., 1995, Visual feature integration and the temporal correlation hypothesis, Annual Review of Neurosciences, 18:555-586.

Softky, W.R., 1994, Sub-millisecond coincidence detection in active dendritic trees, Neuroscience, 58(1):13-41.

Softky, W.R., 1995, McCulloch-Pitts strikes back: A biophysical interpretation of cortical neurons as sub-millisecond binary devices, Mathematics and Computers in Simulation, 40:71-79.

Softky, W.R., 1996, Fine analog coding minimizes information transmission, Neural Networks, 9(1):15-24.

Squire, L.R., 1992, Memory and the hippocampus: A synthesis from findings with rats, monkeys, and humans, Psychological Review, 99(2):195-231.

Steward, O., 1997, mRNA localization in neurons: A multipurpose mechanism? Neuron, 18:9-12.

Tiedge, H., Bloom, F.E., Richter, D., 1999, RNA, whither goest thou? Science, 283:186.

Van der Zee, E.A, and Douma, B.R.K., 1997, Historical review of research on protein kinase C in learning and memory, Prog. Neuro-Psychopharmacol. \& Biol. Psychiat., 21:379-406.

Volfovsky, N., Parnas, H., Segal, M., and Korkotian, E., 1999, Geometry of dendritic spines affects calcium dynamics in hippocampal neurons: Theory and experiments, J. Neurophysiol., 81:450-462.

Wang, X.J., 1999, Fast burst firing and short-term synaptic plasticity: A model of neocortical chattering neurons, Neuroscience, 89(2):347-362.

Wehr, M., and Laurent, G., 1996, Odour encoding by temporal sequences of firing in oscillating neural assemblies, Nature, 384, 162-66.

Whitelam, G.C., and Devlin, P.F., 1998, Light signalling in Arabidopsis, Plant Physiol. Biochem., 36(1-2):125-133.

Zador, A., 1998, Impact of Synaptic Unreliability on the Information Transmitted by Spiking Neurons, American. 